\title{
Feature-positive and feature-negative discrimination learning maintained by negative reinforcement in rhesus monkeys
}

\author{
D. F. McCOY and D. M. YANKO \\ University of Kentucky, Lexington, Kentucky \\ and Air Force Aerospace Medical Research Laboratories, Wright-Patterson Air Force Base \\ Dayton, Ohio
}

\begin{abstract}
Rhesus monkeys were trained to press panels to escape or avoid shock. Escape and avoidance training were combined factorially with feature-positive (FP) and feature-negative (FN) discriminations. In the FP situation, S+ trials were characterized by one red and two green panel displays and S- consisted of an all green panel array. In the FN arrangement, S+ and S- arrays were reversed. When compared properly, FP discriminations were learned more readily than FN discriminations in both escape and avoidance training conditions. Positive tracking was associated with FP discriminations, and feature avoidance characterized the FN tasks. These data suggest that in primates, feature responses (i.e., tracking behaviors) are more a function of the operant contingency than of the Pavlovian correlation.
\end{abstract}

An interesting phenomenon in the area of discrimination learning, and one which has attracted increasing amounts of experimental attention, is the featurepositive effect, FPE, first described by Jenkins and Sainsbury (1969). Consider a go/no-go problem in which an animal is required to differentiate between a white stimulus and a white stimulus with a dot superimposed on it. Whether the subject learns the discrimination is apparently a function of which of these stimuli is designated as $\mathbf{S}+$ and which is $\mathbf{S}-$. If the animal is reinforced for responding to the stimulus containing the dot, or feature, but reinforcement is not available for responding to the blank white key, the condition is labeled feature positive (FP), and discrimination learning appears rapidly. However, in the reverse arrangement, in which the stimulus containing the feature appears exclusively as $\mathrm{S}-$ and the blank white stimulus appears as $\mathrm{S}+$, learning is considerably more difficult. As a matter of fact, this feature-negative (FN) task is frequently characterized by a complete absence of learning. It is this asymmetry in learning FP discriminations relative to FN discriminations which constitutes the FPE.

This research was supported by Laboratory Director Funds appropriated to the Aerospace Medical Research Laboratories at Wright-Patterson AFB, Dayton, Ohio. We are indebted to the personnel of the Veterinary Sciences Division of AMRL for their assistance. Special thanks are extended to Richard Taylor for his reliable assistance in handling the animals, and for the interest and enthusiasm he maintained throughout this study. We are also grateful to Jim Miller, Charles Edwards, and Mike Bardo for their helpful comments. Reprints may be obtained from D. F. McCoy, Department of Psychology, University of Kentucky, Lexington, Kentucky 40506.
Although the FPE has usually been demonstrated with pigeons pecking illuminated keys (e.g., Jenkins \& Sainsbury, 1970), it is by no means confined to that situation. Indeed, FPEs have been obtained with monkeys pressing three-panel arrays (Pace, McCoy, \& Nallan, 1980), with rats pressing illuminated disks (Crowell \& Bernhardt, 1979), and with cats using tone stimuli (Diamond \& Goldberg, 1962). FPEs have also been reported with children (Sainsbury, 1971) and with adults (Newman, Wolf, \& Hearst, 1980).

The apparent generality of the FPE is limited in one sense because all of the above-mentioned studies were based on positive reinforcement paradigms. The only experiment that was concerned exclusively with the FPE and aversive control was one reported by Reberg and LeClerc (1977). Using a conditioned suppression procedure, they were able to demonstrate that suppression developed more readily when the stimuli predictive of shock contained the distinctive feature than in the reverse situation, where the feature predicted the absence of shock. Since the discrimination itself involved a purely Pavlovian paradigm, and since it was impossible to observe the subject's reaction to the feature per se, it is not immediately clear how one can compare this procedure to the standard appetitively controlled operant situation. The present experiment attempted to alleviate this problem by examining FP and FN discrimination learning within the context of a negative reinforcement procedure.

An especially interesting aspect of the FPE is what Hearst and Jenkins (1974) labeled sign tracking. This concept refers to the observation that subjects ex- 
posed to FP discriminations tend to direct their responses exclusively toward the feature (positive tracking), while those given FN discriminations avoid the feature (negative tracking) and respond only to the common elements of the display. In the context of the standard positive reinforcement situation, it is not altogether clear why this should happen. On the one hand, it is possible that behavior is "steered" toward the feature, and that directed responses are evoked simply because the feature is the most reliable predictor of reinforcement, that is, a Pavlovian process (Hearst \& Jenkins, 1974). On the other hand, it also seems possible that the animals are sensitive to the operant contingency, and that they respond to the feature on this basis. The negative reinforcement paradigm should shed some light on this issue. Consider, for example, a FP escape situation: The feature is a reliable predictor of shock, and one would, on Pavlovian grounds, predict a withdrawal response (cf. LeClerc \& Reberg, 1980). On the other hand, the same feature is also predictive of a negative reinforcement contingency. On this basis, subjects might tend to track it positively. Consistently, a FN-escape animal might be expected to track positively because of the Pavlovian correlation but negatively if the operant contingency prevails. In the avoidance paradigm, the symmetry between the feature and shock presence or absence is removed, and, accordingly, the predictions are less specific. This is true because in the FP-avoidance situation, the feature is predictive of shock only in the absence of an avoidance response, whereas in the $\mathrm{FN}$-avoidance arrangement, the feature always predicts no shock, as it does in the FN-escape situation. Nevertheless, feature responses here, which parallel those in the analogous escape situations, might tend to indicate the degree to which similar processes are operative in the avoidance and escape situations.

\section{METHOD}

\section{Subjects}

Sixteen female rhesus monkeys weighing between 18 and $24 \mathrm{~kg}$ served as subjects. All were experimentally naive. The animals were housed individually and maintained on ad-lib food and water. Light-dark alternations occurred at 12-h intervals in the colony room.

\footnotetext{
Apparatus

During experimental sessions, the animals were restrained at the neck and waist in a standard primate chair. Their feet were placed into small shoes equipped with brass sole plates designed to deliver shock. At the beginning of each experimental session, the restrained animals were placed into a $162.5 \times 82.6 \times 64 \mathrm{~cm}$ sound-insulated chamber. The intelligence board contained three horizontally arranged translucent panels measuring $8 \mathrm{~cm}$ sq. The panels were spaced $8.8 \mathrm{~cm}$ apart and located at about eye level of the subjects. Each of the panels could be back-illuminated with green, red, or white light. A closed-circuit television system was used to monitor the subject's behavior, and a Grason-Stadler shock generator provided shock, which was delivered at a rate of $10 / \mathrm{sec}$. The chamber was also equipped with a white-noise gen-
}

erator, which provided an 80-dB output sufficient to mask extraneous sounds. A Cromemco computer was used to program and record experimental events.

\section{Procedure}

Initial training was conducted with an all-white panel display. Shock intensity was adjusted on an individual basis so that it was minimal but sufficient to maintain consistent escape behavior. The intensity ranged between 4 and $7 \mathrm{~mA}$. The animals were first trained to press any one of the three illuminated panels in order to terminate randomly scheduled shock. In addition to terminating shock, each escape response produced a 1-sec offset of the stimulus array. This preliminary training was completed within three sessions.

The animals were next assigned to one of four treatment groups: FP-avoidance, FP-escape, FN-avoidance, or FN-escape. In each of these conditions, two types of stimulus arrays were presented. Feature trials consisted of two green panels and one red panel. On these trials, the red feature was equally likely to appear in any of the three possible positions. Nonfeature (common) trials contained three green panels. In the FP conditions, feature trials were labeled $\mathbf{S}+$ because they were predictive of shock and a response contingency. S- trials were characterized by the illumination of three green panels, which signaled neither shock nor contingency. For the FN treatments, these stimulus relationships were reversed; that is, common trials were $\mathbf{S}+$ and feature trials were $S-$.

In the FP-avoidance condition, the avoidance interval on $\mathrm{S}+$ (feature) trials was $5 \mathrm{sec}$. During this time, a response on any of the panels cancelled shock and terminated the display. A response which did not occur until after the 5-sec interval terminated both shock and the stimulus display. On S- trials, the all green panel display remained illuminated for $10 \mathrm{sec}$ regardless of the number of responses that occurred. In the FN-avoidance condition, responses on any of the panels during common $(\mathbf{S}+)$ trials avoided shock and terminated the display if they occurred within the first $5 \mathrm{sec}$. On feature ( $\mathrm{S}-$ ) trials, the stimuli remained illuminated for 10 sec. FP-escape trials were identical to those of the FPavoidance condition except that responses during the initial $5 \mathrm{sec}$ of S+ trials had no consequences. At the end of the 5-sec interval, shock began and the first panel press terminated both shock and the stimulus display. On S- trials, shock was not scheduled and the panels remained illuminated for a full $10 \mathrm{sec}$. In the FN-escape condition, the contingencies were the same, but the stimuli signaling these events were reversed. In all conditions, the intertrial intervals were variable, ranging between 10 and $60 \mathrm{sec}$.

Experimental sessions were run on weekdays. Each session was composed of 48 trials, half feature trials and half common trials. The order of stimulus presentations was mixed, but with the restriction that a maximum of three presentations of a given configuration could occur consecutively. Training was carried out until a maximum of 30 sessions was run, or until the discrimination criterion was met. The discrimination criterion was defined as six consecutive sessions with less than $10 \%$ deviation in the discrimination ratio.

\section{RESULTS}

Responses to $\mathbf{S}+$ and $S$ - were recorded separately. It will be recognized, however, that $S$ - duration was fixed $(10 \mathrm{sec})$, whereas $S+$ duration was under the control of the subject. Because S-duration was always considerably longer than $\mathrm{S}+$ duration, multiple responses could occur to $S$ - while only a single response was possible to $S+$. These differences could alter the character of the discrimination picture. In order to adjust for these discrepancies, stimulus rates were calculated so as to take into account the relationship between response frequency and stimulus 
duration. In general, these rates were calculated as follows: number of responses to the stimulus/total stimulus duration. Discrimination ratios (DRs) were then calculated for each session by dividing $S+$ rate by the combined $S+$ and $S-$ rates. Using these DRs, a $2 \times 2$ ANOVA was applied to the sessions-tocriterion data. Escape animals reached criterion significantly faster than did avoidance animals $[F(1,12)$ $=8.98, \mathrm{p}<.05]$. There was a slight, but nonsignificant, tendency for FP animals to reach criterion faster than $F N$ animals $[F(1,12)=3.02, p>.05]$. The interaction did not approach significance.

The left panel of Figure 1 presents the overall DRs for the four treatment groups during their final six sessions. A $2 \times 2$ ANOVA performed on these data revealed a clear FPE $[F(1,12)=10.71, p<.01]$. Escape-trained animals performed better than avoidance animals $[F(1,12)=12.92, p<.01]$, and the interaction was also significant $[F(1,12)=18.03$, $p<$ .01]. A simple main effects analysis indicated that this interaction resulted from the fact that the difference between FP and FN performance was significant in the avoidance condition $[F(1,12)=19.17$, $p<.01]$ but not in the escape condition. The overall tracking ratios during these same sessions appear in the right panel of Figure 1. Tracking ratios were computed by dividing the number of responses to the feature by the total number of responses occurring on feature trials. FP animals responded to the feature significantly more than did their FN counterparts, who responded to the feature at about a chance level $[F(1,12)=15.51, p<.01]$. Neither the main effect of escape versus avoidance nor the interaction was significant.

The overall discrimination superiority in the escape relative to the avoidance condition seen in Figure 1 is probably due to the fact that escape behavior was maintained by the presence or absence of shock rather than by the discriminative stimuli signaling these events. Obviously this is not true for the avoidance treatments. In order to examine the degree to which stimulus control was actually present in these situations, discrimination and tracking performances were calculated separately for the 5-sec stimulus interval preceding shock. These data appear in Fig-
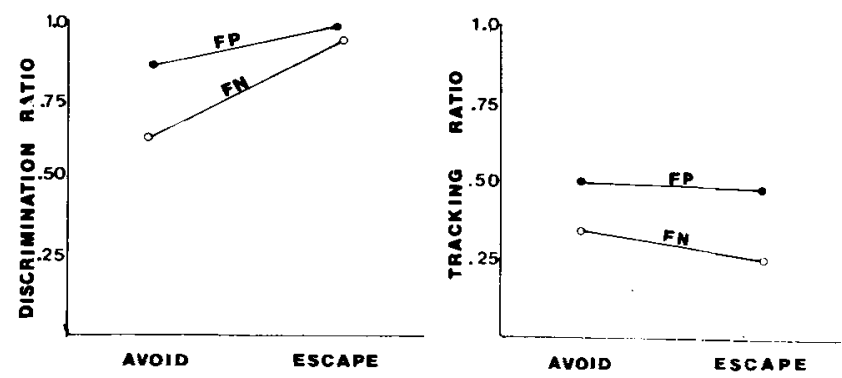

Figure 1. Discrimination ratios and tracking ratios for overall performance.
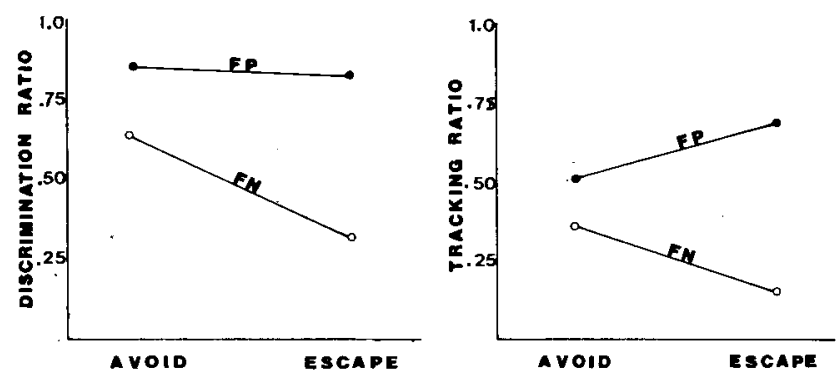

Figure 2. Discrimination ratios and tracking ratios during the preshock stimulus interval.

ure 2. As expected, the data for the avoidance treatments remained essentially unchanged, while those from the escape conditions were altered considerably. A $2 \times 2$ ANOVA applied to the discrimination performance (left panel) supported the impression that the overall superiority of the escape treatment present in Figure 1 was now absent. However, a robust $F P E$ was again present $[F(1,12)=16.35, p<.01]$, along with a significant FPE $\times$ training condition interaction $[F(1,12)=11.99, p<.01]$. It can be seen, however, that the nature of the interaction was quite different from that in the original analysis, namely, a larger FPE in the escape condition. A simple main effects analysis supported this assertion by revealing significant FPEs in both the avoidance $[F(1,12)=$ $7.16, p<.05]$ and the escape conditions $[F(1,12)=$ $16.03, \mathrm{p}<.01]$. More importantly, subjects in the FN-escape situation performed more poorly than those in the $F N$-avoidance condition $[F(1,12)=$ $10.19, \mathrm{p}<.01]$, whereas there was no difference in the performance of FP-avoidance and FP-escape animals.

The change in discrimination performance for the escape groups in the preshock stimulus interval was accompanied by an altered pattern of tracking as well (left panel, Figure 2). Responses to the feature increased in the FP-escape condition and decreased in the FN-escape condition, while those of the respective avoidance groups remained unchanged. The results of an ANOVA applied to these data were generally consistent with the pattern described above, namely, a nonsignificant overall difference between escape and avoidance tracking, but significantly more tracking in the FP conditions $[F(1,12)=23.24$, $\mathrm{p}<.01]$ and a pronounced FPE $\times$ training condition interaction $[F(1,12)=10.13, p<.01]$. Further analytic comparisons involving a simple main effects analysis indicated that FP animals tracked more than FN animals in both the avoidance condition $[F(1,12)$ $=4.88, p<.05]$ and the escape condition $[F(1,12)$ $=14.23, \mathrm{p}<.01]$. Of special interest is the finding that FP-escape animals tracked significantly more than FP-avoidance animals $[F(1,12)=4.91, \mathrm{p}<.05]$, whereas FN-escape subjects tracked significantly less 
than their $F N$-avoidance counterparts $[F(1,12)=$ $10.67, \mathrm{p}<.01]$.

\section{DISCUSSION}

The present results extend the generality of the FPE to the context of negative reinforcement. When properly compared, performance on both discriminated escape and avoidance tasks is enhanced when the distinctive feature appears on $\mathrm{S}+$ trials rather than on S- trials. This relationship is true as a total performance phenomenon, but it is evidenced even more clearly when the behavior is examined in the presence of the discriminative stimuli during the preshock interval alone. It should be noted, however, that this generalization pertains to terminal performance rather than to rate of learning.

Reberg and LeClerc (1977) reported an FPE with a conditioned-suppression procedure; however, their subjects did show some learning in the FN situation. In the present experiment, strong FN learning appeared only in the escape condition when responses were probably controlled by the presence or absence of shock rather than by the accompanying discriminative stimuli. When discrimination behavior was evaluated in terms of the signaling stimuli only, little learning was present in either the FN escape or the FN avoidance situation (DRs of 64 and 33, respectively). To this extent, then, the present data are comparable to those typically reported from the positive reinforcement paradigm (e.g., Jenkins \& Sainsbury, 1969, 1970).

The present tracking data are of special interest. Reliably more responses occurred to the feature when it signaled shock $(\mathrm{S}+)$ than when it signaled the absence of shock (S-). This relationship is somewhat confounded in the avoidance condition, since, by definition, a successful avoidance response precludes shock. It is not true in the escape situation, however, since all $\mathrm{S}+$ trials signal shock. In this connection, it will be recognized that when the preshock interval alone is considered, tracking became more pronounced in the FP-escape condition, while avoidance of the feature became apparent in the FN-escape situation.

The tracking data warrant additional consideration for other reasons. In the first place, the tendency to approach or avoid the feature was compromised by strong position preferences which were present in every animal studied. Indeed, 15 of the 16 animals exhibited a marked preference for the center panel. The other animal responded mainly to the right panel. Between $86 \%$ and $98 \%$ of all responses occurred on the preferred panel. Given this strong position preference, it is impressive that systematic responding was directed toward the feature at all. Yet, FP-escape animals tracked at about $\mathbf{7 0 \%}$, which is tantamount to concluding that virtually all nonpreferred panel responses involved the feature. FN-escape animals, on the other hand, evidenced a decided avoidance of the feature despite the fact that it appeared on the preferred panel $33 \%$ of the time. Thus, responses on nonpreferred panels probably reflect an avoidance of the feature present on the preferred panel. These strong position preferences may explain why the positive and negative tracking observed here were not as robust as those typically seen in the positive reinforcement situation (cf. Pace, McCoy, \& Nallan, 1980).

The tracking data may also have implications which extend beyond the mere demonstration of an FPE produced by negative reinforcement. According to a Pavlovian interpretation, the tendency to approach or avoid the feature will be a function of the type of event with which it is correlated. It is for this reason that Hearst and Jenkins (1974) argued that, in the appetitive situation, the responses of the FP subjects are steered towards (and elicited by) the feature. Similar logic could be applied to a study by LeClerc and Reberg (1980), in which it was shown that rats tend to avoid contact with stimuli that signal the occurrence of shock in a Pavlovian paradigm. From this perspective, one would expect, in the present situation, negative tracking by FP subjects and possibly positive tracking by FN subjects. Yet, exactly the opposite relationship emerged. More feature responses were found in both FP conditions than in their respective FN conditions. This effect was especially strong in the escape condition in which the FP animals responded even more readily to the feature, while the FN animals demonstrated marked feature avoidance. Taken together, these findings suggest that tracking may be more than the reflection of a simple elicitation process. It seems that feature tracking is not just elicited by the type of stimulus event that the feature predicts, but rather that tracking occurs because the feature is the informative aspect of the situation; that is, because the feature predicts the significant behavioral events in the situation.

Despite the above-mentioned considerations, the Pavlovian contribution to this experiment cannot be ignored. Indeed, the predictive relation between the feature and shock implies such a process. In addition, we observed the importance of Pavlovian processes very early in training when panel responses were being shaped. Initially, the three-panel array was illuminated only during the preshock interval. It became immediately clear, however, that this procedure resulted in avoidance (i.e., withdrawal) reactions rather than the required approach responses. It was for this reason that the procedure was changed so that during response shaping, the panels remained illuminated continuously, except for a brief offset following a correct response. Notwithstanding, it cannot be denied that when the FP animals did press panels later in training, they tended to direct their responses to the feature itself, and that these re- 
sponses were not those originally elicited by the stimuli that had signaled aversive events. These approach responses required by the operant contingency, tended to override the "more basic" tendencies to avoid the feature stimuli. In this connection, it will be recalled that the shock intensity presently employed was minimal. It is possible that with increased shock intensity a different feature-response relationship would have emerged. Finally, it is also possible that the ability to override more reflexive types of behaviors represents a more cognitive approach which the primate brings to the FP problem, and that these tendencies may be absent in nonprimates.

\section{REFERENCES}

Crowell, C. R., \& Bernhardt, T. P. The feature-positive effect and sign-tracking during discrimination learning in the rat. Animal Learning \& Behavior, 1979, 3, 313-317.

Diamond, I. T., Goldberg, J. M., \& NefF, W. D. Tonal discrimination after ablation of auditory cortex. Journal of Neurophysiology, 1962, 25, 223-235.

Hearst, E., Jenkins, H. M. Sign tracking: The stimulus- reinforcer relation and directed action. Austin, Tex: Psychonomic Society, 1974.

Jenkins, H. M., \& Sainsiury, R. S. The development of stimulus control through differential reinforcement. In N. J. Mackintosh \& W. K. Honig (Eds.), Fundamental issues in associative learning. Halifax: Dalhousie Press, 1969.

Jenkins, H. M., \& Sainsbury, R. S. Discrimination learning with the distinctive feature on positive or negative trials. In D. I. Mostofsky (Ed.), Attention: Contemporary theory and analysis. New York: Appleton-Century-Crofts, 1970.

LeCle RC, R., \& Reberg, D. Sign tracking in aversive conditioning. Learning and Motivation, 1980, 11, 302-317.

Newman, J., Wolf, W. T., \& Hearst, E. The feature-positive effect in adult human subjects. Journal of Experimental Psychology: Human Learning and Memory, 1980, 6, 630-650.

Pace, G. M., McCoy, D. F., \& Nallan, G. B. Feature-positive and feature-negative learning in the rhesus monkey and pigeon. American Journal of Psychology, 1980, 93, 409-427.

Reberg, D., \& LeClerc, R. A feature positive effect in conditioned suppression. Animal Learning \& Behavior, 1977, 5, 143-147.

SaINSBURY, R. S. Effects of proximity of elements on the featurepositive effect. Journal of the Experimental Analysis of Behavior, 1971, 16, 315-325.

(Manuscript received March 28, 1983; revision accepted for publication June 28,1983 .) 